\title{
PERSONALIZED WELLNESS IN SMART OFFICES
}

\author{
Vincenza Carchiolo ${ }^{1}$, Alessandro Longheu ${ }^{2}$, Sergio Barbera ${ }^{3}$ and Pietro Di Tommaso ${ }^{3}$ \\ ${ }^{1}$ Università degli studi di Catania - Dipartimento di Matematica ed Informatica, Italy \\ ${ }^{2}$ Università degli studi di Catania - DIEEI, Viale A. Doria 6, Catania, Italy \\ ${ }^{3}$ Bax Energy, Via Sclafani, 40/b, 95024 Acireale CT, Italy
}

\begin{abstract}
IoT and AI are endorsing a historic change in many fields, leading to a smarter world. This also occurs in building management, where energy consumption, climate control and wellness for people are all factors to ve addressed. In this work, the smart office solution deployed inside BAXEnergy is introduced. It aims to reduce energy consumption, improve the quality of daily life while addressing internal security issues.
\end{abstract}

\section{KEYWORDS}

Natural Language Processing, Smart Buildings, Internet of Things, Smart Offices

\section{INTRODUCTION}

In the last decades, the advances in computer-based technologies allow machines to operate almost without human intervention. The main purpose of this technologies is to enable computers to autonomously learn from, decide what, and even forecast the actions to carry out for a particular task, according to the external context.

Some of the most important research areas involved in this trend are the Internet of Things (IoT) and Artificial Intelligence (AI). IoT [Lin et al. 2017] [Atzori et al. 2017] refers to the interconnection of ubiquitous electronic devices each other to gather, monitor, control and transfer information over the network. The main purpose of IoT is to collect the huge amount of data generated by those devices in a given scenario, then extracting and leveraging this information to promote useful and innovative everyday applications and services. On the other hand, AI is a discipline focused on instructing a computer to ultimately think and learn as humans [Lake et al. 2017]. Both IoT and AI endorse what is nowadays known as "smart" world, i.e. systems that incorporate the concepts of self-learning, analysis and actuation to forecast autonomously the best possible decision. A context where getting smarter will determine great benefits is that of Smart Buildings [Bolchini et al. 2017], both residential (smart home) and commercial (smart office), whose relevance is mainly due to their contribution to worldwide energy consumption (about 30-40\%) [Chen et al. 2009], actually the second largest energy-consuming sector after transportation. Both areas have some common objectives when it comes to using smart systems, such as reducing energy consumption and improving the quality of daily life, according to users' preferences [Shaikh et al. 2018]. Wellness includes several factors, as setting correct values for temperature and humidity, achieving good quality for air and lighting as the most basic, but also ensuring psychological and physical comfort (for instance having some break during working hours), promoting happiness and positive attitude and keeping constructive personal relationships (both with colleagues and bosses).

While sustainable planning of working activities can be achieved by adopting effective ERP software, factors cited in the previous paragraph are sometimes difficult to address, although they play a relevant role in stress prevention, which is the second most frequent work-related health problem in EU countries, with an estimated cost of 617 billion a year [EU, 2018] [Alberdi et al., 2018]. At the same time, preserving worker's everyday life quality positively affects company performance and productivity. This paper presents the Smart Office solution inside BAX Energy workspaces [BAX, 2019], operating in a business environment, whose objectives are to reduce energy consumption, to improve the quality of daily life as specified above, also addressing internal security issues. The system benefits from the integration of IoT and AI-related techniques as Natural Language Processing (NLP) and facial recognition. IoT is "the key for the control and surveillance of Intelligent Buildings" [Plageras et al. 2017], one of the basic brick in Smart Cities development, whereas AI 
is first used to properly gather all working-related user data transparently, so it can then allow performing environment adjustments in accordance with user-preferences.

Facial recognition, in particular, increases significantly the internal workspace security because it differs from standard system based on cameras monitored by security guards and/or personal badges distributed to employees. This technology allows instructing a system to recognize the identified person and react depending on the verification result. Moreover, facial recognition can be exploited to detect different situations such as the level of comfort of a person or his emotional state. The same goes for NLP techniques: such feature can be very useful for simple operations that otherwise would probably be handled by the administration area of a company. Thanks to these tools, whenever a user interacts with the system he/she is initially identified via his/her face, then the system takes care about that user either by exploiting information about his past behavior and response stored in users profile, or it can react to specific request the user provides using GUI or voice commands (processed by NLP tools) in an intuitive and interactive fashion. The proposed system "takes care" about each user in different ways, both proactively and reactively, as for instance offering the best suitable seat for him/her based on position and/or neighborhood, or tailoring environmental parameters (lights, air, humidity), checking seat reservation (by recognizing whether the person being seated is the right one or not) or finally dynamically detecting users mood and reacting to provide him/her with best possible working conditions. These features are implemented via an architecture that includes sensors, cameras, microphones (all embedded as IoT devices), a data-gathering module to convey all users profiles and a web application to manage the whole system.

In the following sections, we first introduce some existing works about smart buildings (Section 2), while in section 3 the system features and architecture are discussed. In section 4 some implementation details are presented, while conclusive remarks and future works are briefly outlined in section 5 .

\section{RELATED WORK}

There are several solutions available in the literature about smart buildings, and in particular for what concern smart offices. Generally, both competing issues are addressed, i.e. there are works focusing on consumption reduction [Choi et al. 2015] [Rottondi et al. 2015], and others where wellness on work time is promoted [McDuff et al. 2012] [Kiyokawa et al. 2012] [Stankevic et al. 2011]. Some solutions are hardware-based, that is they provide smart devices to be installed throughout the workspace. Sometimes sensors data are displayed by a specific dashboard provided by the same company, and the development of personalized interfaces (useful in case of particular needs) may be also supported. Other solutions are software-based, i.e. the software application is used to connect smart devices in order to exploit data that have been collected. In such cases, the dashboard included in the software comes with many (possibly customizable) functionalities.

Most smart office solutions offer similar features: indoor environmental measures (temperature, noise, humidity, and others), metering measures and occupancy data. What makes the difference is the quality of those provided data and the involvement of AI technologies within the system. The question of which and how worker-related data should be collected has been debated from several points of view. For what concerns the type of data to monitor, some applications [Basnayake et al., 2015] focus on user comfort, safety, and enhanced energy performance, while others also consider psychological related information, as in [Muoz et al. 2018] and [Alberdi et al. 2018]; here we aim to address both types of user data, to promote total wellness.

In past years, data was extracted from self-reported questionnaires [Lang 1980] [Hart and Staveland 1988], but this approach requires explicit user cooperation and may be biased by his/her emotional state, resulting in possible deceptive datasets. To overcome these limitations, recent techniques are non-intrusive and this lead to the massive use of smart devices and therefore to IoT, also involving Big-Data and Cloud Computing [Plageras et al. 2017], the former research area being useful for the high volume of data collected, and the latter to cope with storage capacity, scalability and reliability. We fully comply with this approach, in particular within the Bax Energy workplace a set of smart devices able to track environmental parameters like temperature, noise, humidity, $\mathrm{CO}$, lighting and VOCs (Volatile Organic Compounds) is adopted. Moreover, we also have specific devices that track the presence of a person thanks to a proprietary algorithm. Each of that is installed underneath a work-seat in order to know if a person is occupying it. Their algorithm exploits the variation of the temperature due to the presence of a seated person and considers several parameters to do that as current environmental temperature, human temperature, and any other eventual temperature source (e.g. under a desk 
a tower PC may increase the temperature when powered on), in order to avoid that any possible occluding item could be misunderstood as if a person were seated.

In addition, if some person moves his/her legs under the table, the proprietary device does not toggle its state thus avoiding false tracking information. Other solutions also implement such fine-grained detection, e.g. in [Akbar et al. 2015] authors introduced the "standby" state in addition to "present" or "absent" to model situations when the worker lefts his seat for small breaks. Moreover, the support for user personal profile we propose can be also observed in other works, e.g. in [Muoz et al. 2018], an emotion aware automation platform that allows creation and configuration of personal automation rules are proposed.

From a broader perspective, in [Bolchini et al. 2017] a framework for data gathering is presented, addressing several dimensions as the campaign time span, the time granularity for data collection, the sampling strategy, final goals etc., whereas in [Lilis et al. 2017] authors point out that the significant assets of current automation tools should not be discarded rather an integration with emerging innovations (as IoT) is encouraged. Considering the way of displaying the workspace map, most of the smart building applications allow displaying a custom map do that using CAD software. This approach guarantees a detailed three-dimensional plan of the monitored building, though such maps often require proper hardware to be rendered. CAD drawings can hardly be rendered by mobile devices. This involves the need to have separated applications if a user wants to access to smart building systems by smartphone or tablet.

For this reason, we preferred to design a web application as a user interface of our smart building management system. A web application provides a less detailed building map but it needs fewer hardware requirements so most devices (laptops, smartphones and tables) can easily do it. Moreover, a web application can be accessed anywhere, instead of software that needs to be installed on a specific machine.

Another aspect that led us to use web-based technologies is that the workspace to monitor is built in a single floor, hence a detailed two-dimensional image has been used to represent the office planimetry so that all browsers can easily render it. Recent studies discuss AI capabilities involved in smart building systems for analyzing sensors data to forecast some deterministic behavior. Some example concerns the possibility for the system to understand when a voltage overload is going to be encountered so that a blackout can be avoided. This kind of AI capabilities is not though present in any existing smart building solution and is not considered in this work. The question of finding a trade-off between energy consumption and users requirements can be modeled as a multi-objective optimization, for which several techniques exist [Nguyen et al. 2014]. We exploit AI techniques also for the data gathering phase before their processing, in particular to extract information from sensors as well as from camera/microphones about user current state (satisfactory or discomfort) and preferences.

\section{FROM SMART BUILDINGS TO SMART WORKPLACES: CASE STUDY}

The smart building system introduced in this paper can be better characterized as a smart office proposal, indeed in addition to classical energy consumption management and environmental control, we also aim to put the worker in the best mental and physical condition to increase his/her productivity. This is achieved using GUI, as well as microphones and cameras as input devices through which the user can be recognized and then express his/her preferences; for instance, he/she can select a seat with a specific landscape (whenever possible), or near/far from some colleague. Moreover, the system also exploits past choices to proactively suggest the option according to personal profiles. In the following, we first provide an overview of system functionalities, with details about face recognition and NLP, then introducing the overall system architecture.

\subsection{System Functionalities}

This system aims to fully cover the management of a workspace exploiting AI and IoT technologies. There are several departments to take care of in a generic workspace: administration, internal security, management of indoor space and others.

Considering the internal security, most companies require to automatically identify persons to restrict access in the office just to the expected ones. The first category of authorized persons are the employees or, in general, internal staff. A simple case presents employees that are identified and verified by a facial recognition software at the main entrance of the office. The same procedure can be applied within the office for specific 
restricted areas. Of course, we first need to train the software to correctly identify employees, for this analysis we limit the recognition to a known set of persons.

Another use case of internal security to be addressed here refers to planned appointments. Typically, scheduled dates are managed directly by the administration and/or HR departments; the idea is to implement a specific assistant based on NLP technology that manages all incoming dates. This assistant is programmed to welcome the guest, ask for his name and the reason for the appointment. For example, the assistant welcomes a person that is going to have an interview, hence it searches for his name and the reason for the visit among scheduled dates: if none has been found, the assistant considers this visit as unexpected and contacts someone of company administration, otherwise it leads the guest to a specific area where to go (of course, the assistant actions may vary depending on company internal policy). Another use case studied here is specific for open workspaces where more companies share the business area. In such kind of offices there are no preassigned workstations to employees, so it required a daily booking process that manages them in the whole office. Moreover, to guarantee that a specific seat is occupied by who requested it, a facial recognition system can check who is going to seat: if the person is the one expected, the system can electrically power the desk and adjust the lighting.

In addition to the above features, two games have been deployed to exploit the capacity of a trained computer to identify/recognize what it sees: FaceChallenge and Guess your origins. Face-Challenge asks users to emulate some random expression among sadness, anger, happiness, and seriousness. Each time the user does it gains points. Guess your origin tries to guess the age, the gender and the country of origin of the user. The interface applies a colored border (pink or light-blue, depending on users gender) around the users face and guess his/her age and native country, analyzing his/her somatic features. Often, the best way to show something is by giving the possibility to test it or play with it; that way those two games have been designed in this smart office management system.

\subsection{Assistive Technologies: Face Recognition and NLP}

Facial recognition mechanism adopted in the work here described is based on Convolutional Neural Network (CNN) [Zheng, 2018] [Zeiler et al, 2014]. A simple CNN is a sequence of layers, and every layer of the network transforms one volume of activations to another through a differentiable function. In deep learning, each level learns to transform its input data into a slightly more abstract and composite representation. For example, in a face recognition application, the raw input may be a matrix of pixels; the first representational layer may abstract the pixels and encode edges; the second layer may compose and encode arrangements of edges; the third layer may encode a nose and eyes; and the fourth layer may recognize that the image contains a face.

In facial recognition, the main subject of images passed as input to a CNN is the face. First of all, we need to know how to differentiate between a basic face and the rest of the background. Facial recognition software is based on the ability to recognize a face and then measure the various features of the face and of the person, such as gender, age, and sentiment. Every face has numerous, specific landmarks that make it distinguishable from others. There are approximately 80 landmarks on each human face, as the distance between the eyes, the width of the nose, depth and curve of the eye sockets, the shape of the cheekbones, the length of the jawline and so on. Here we exploit facial recognition in two different scenarios. In verification, an image is matched to only one of those in the database to verify the subject is who he says to be. In identification, the image is compared to all images in the database resulting in a score for each potential match to identify the subject.

Natural Language Processing (NLP) [Jackson and Moulinier 2007] here is applied to build a dialogue system and also to collect information about user preferences and satisfaction/unsatisfaction. Today dialogue systems are being widely adopted, as Google assistant (https://assistant.google.com/), Windows Cortana (https://www.microsoft.com/enus/cortana) and Amazon Alexa (https://developer.amazon.com/it/alexa); in the context of Bax Energy, a proprietary solution is adopted. Moreover, to detect users satisfaction (promoting his/her wellness), sentiment analysis is also performed. Also known as opinion mining [Medhat et al. 2014] [Goncalves et al. 2013], Sentiment analysis exploits NLP, text analysis and computational linguistics to extract subjective information, as the mood of the people regarding a particular product or topic; it is essentially the classification problem of labeling a given text as positive, negative or neutral. Sentiment analysis has been applied in a variety of scenarios, e.g. [Carchiolo et al. 2015] [Diakopoulos and Shamma 2010]; in this case, the source text is captured with a microphone and the topic is users wellness. 


\subsection{System Architecture}

The system can be modeled as shown in Figure 1; it includes IoT devices, the web application, databases for both and the smart management system. The IoT devices block includes sensors, cameras, and microphones; sensors are distributed throughout the workspace to measure constantly the environmental conditions: temperature, humidity, $\mathrm{CO} 2$, power consumption and lighting. Thanks to sensors, the system can monitor indoor parameters, display them in through the web application and if some value exceeds predetermined thresholds it can also adjust the related physical quantity by properly triggering heat/electricity/airflow management systems.

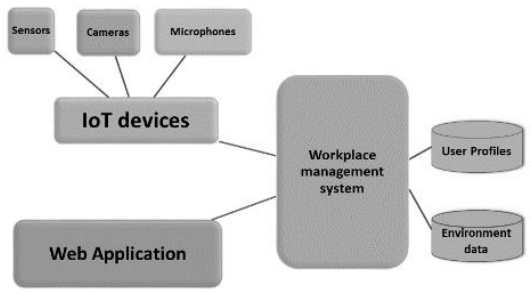

Figure 1. Back-end overview

Note that, in case such management systems cannot be directly controlled (as it frequently occurs in existing workplaces where full integrated environment control maybe not present), the system anyway display messages on the web application to manually change the conditions.

Moreover, meter sensors also allow monitoring power consumption of the office to check any power losses or identify excessive consumption of some devices. Cameras are used to detect whether a work seat is available or not, while microphones allow users to interact in a user-friendly way with the management system, in addition to the GUI provided by the web application. Data collected are stored in two databases, one devoted to users profiles and the second user for environmental data; data are steadily gathered, so the current state about the workplace as well as about workers wellness can be dynamically updated.
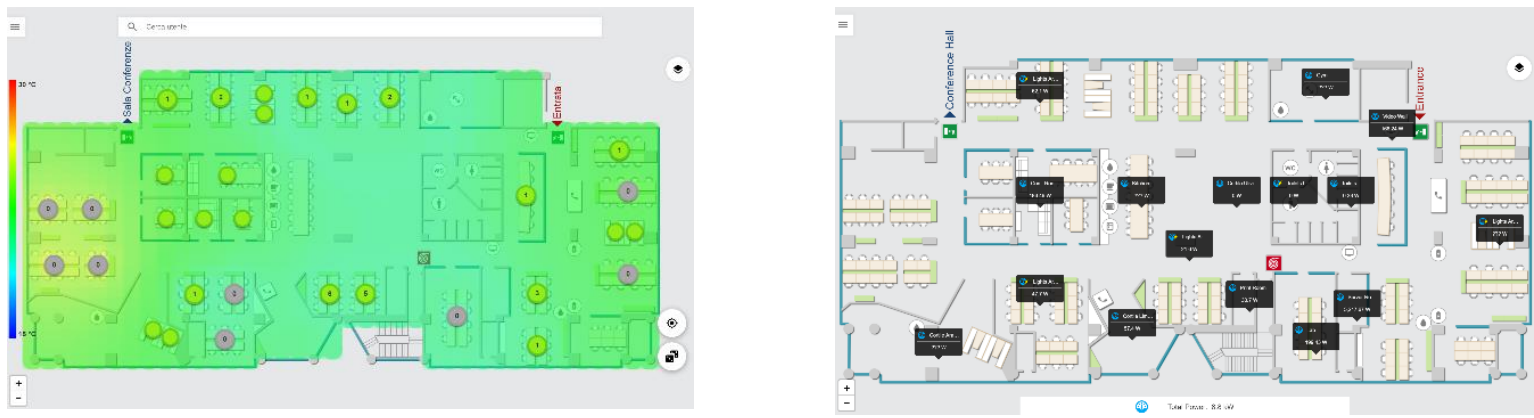

Figure 2. Workplace temperature and consumption map

The web application provides a 2-D real-time map information about the workplace, as temperature and power consumption (figure 2); it also helps users in their requests, for instance allowing to select the seat with desired ambient conditions.

\section{IMPLEMENTATION NOTES}

The entire application can be split into two blocks: front-end side, including GUI and the implementation of API used to allow the web application communicate with other services, and the back-end side, where the core of the application is implemented. Back-end side is structured in different services: Security service, Profile service, Core service, OPC service, IEC substation service, AI service. Both sides are hosted on two different Windows Server virtual machines and access is granted to about sixty users simultaneously. There are specific firewall rules configured into the virtual machine hosting the back-end so that it can be queried just by 
front-end virtual machine, whereas functionalities are not available for external access. This increases the security of the proposed smart office management system.

Smart sensors and devices connect with the backend side, sending environmental data and receiving control operations, whereas facial recognition and NLP systems run as a separate module from the back-end since they hold the sole connection with cameras and microphones.

Sensors and devices are proprietary and produced by WiSNAM s.r.l. (https://www.wisnam.com/); installed devices are named AirSenses and PreSenses, the former measuring temperature, lighting, air quality and noise of the environment and the latter tracking the presence of a person seated on a specific work seat. AirSenses have been mounted one per desk, one per meeting room, and some into the common areas. PreSenses have been mounted one per working seat. In the following, the front-end side (web server), as well as each sub-module of the back-end side, are briefly discussed.

The front-end block is hosted into a Windows Server virtual machine and includes the web application, running on an IIS server, that responds to all requests coming from users' browser. The Web Server interacts with each service hosted into the back-end side: it sends the authentication request to the security service, when a user attempts to log into the application; it retrieves users configurations by querying the profile service; it requests all information (e.g. meeting room reservations) to the core service; it requires sensors data and forwards control commands through the OPC service.

The web server is also responsible to render the office map. Depending on the zoom level, the web server renders the office map tiles that can be contained into users' screen with proper office status information. A higher zoom level determines a bigger number of details. To populate the office map with those data, the Web Server must query the Core service to gather all significant information to display.

The Security service manages the user authentication process. When a user attempts to log into the application, his credentials are handled by this service that validates them and gives back an authentication token, if credentials provided are correct. To check the validity of credentials, the security service retrieves users information stored into the main database and compare them with the received ones.

An authentication token is stored by the browser as a cookie; it will be inserted into any further request to prove to other services that those requests are legit. Furthermore, the security service checks specific users permissions within the smart building management system. Users can have less or more privileges than others, e.g. someone can reserve a meeting room on behalf of another one, while some users can't reserve a meeting room at all. Such information is sent back to the web server, during the initialization process of the UI.

Once the user is authenticated, all his/her information is requested to the Profile service, which is responsible for storing and loading settings associated with currently logged in user. Profile service gets users information from the main database of the system and returns it to the web server, so that it can properly populate the application interface.

The Core service returns all information needed to use the smart office management system. To properly render the UI, several data stored in the main database are requested. The office map indeed has to be populated with all desks and meeting rooms that a user can reserve to work. The Core service gets coordinates of those items so that the Web Server can correctly plot them upon the office map, and their reservation calendars, so that the user can see which desks and rooms are still available. Moreover, the Core service returns to the Web Server also the coordinates of all sensors installed around the office and their specifications, so that the user can see in the map where they are positioned and what kind of environmental parameters they monitor

The OPC service [Yao, 2012] handles subscriptions by different clients to sensors data. Communication between clients and the OPC service are based on OPCDA protocol. The Web Server implements internally the OPC client logic. Each time, a user wants to read one or more sensor values, he/she sends a request of subscription for those sensors via the Web Server and the OPC service will receive it.

Several requests can be sent by different users for the same sensor, hence the OPC service groups them by the sensor. Next, the OPC service subscribes to the IEC substation [Matoušek, 2017] for the data requested by users. IEC substation retrieves environmental parameters from sensors and each time one of them changes, it sends them to the OPC service. OPC service is periodically polled by all subscribed clients independently from each other. A client receives back sensors data only if something has changed since its last request. Once data have been received, the user interface is updated with new environmental values. In addition, the user can send control commands by using the UI. For example, he can switch on an office light near his desk. Those commands are sent to the OPC service, then forwarded to the IEC substation that can send the command to the proper smart device. 
The IEC substation service is responsible to get data from sensors and provide them to all subscribed clients. In this architecture, we have just the OPC service as client subscribed to sensors data. IEC substation does not communicate with the Web Server directly and needs to be configured in order to correctly connect to installed sensors and smart devices; typically, this is a back-office work. IEC substation sends data to OPC service exploiting on change pattern, that is, they do not communicate periodically, but only if some parameter monitored by the sensors has changed. The IEC substation also handles control command received by the OPC service. When a control command is performed, the IEC substation sends feedback, so that the user can know whether his/her request has been successfully completed or not.

The AI service is devoted performing calculations on data retrieved from sensors installed in the office. When a user reserves a desk or a meeting room, the Web Server sends ambient parameters retrieved from the OPC service to the AI service. This should be able to forecast environmental conditions in the next hours using current parameters so that it can eventually suggest changing the reservation (this functionality is currently being tested). This is useful for instance when a user reserves a room but the AI service detects that it is too small for the expected number of persons. The AI service is also responsible to monitor meter devices. It subscribes to the OPC service to be informed about power consumption data. Whenever it detects something unusual, e.g. if some device is consuming more than expected, it isolates the device and informs IT company department through a notification system.

The last module is Facial recognition and NLP systems, both belonging to the smart building management system, but they work independently of the other components. They are responsible for company internal security, by exploiting cameras installed all around the office. Cameras monitor the workspace continuously. They send one frame of the stream per 250 milliseconds to the facial identification service. If a face is detected within the analyzed frame, this is sent to the Face API service implemented on Azure cloud.

The Microsoft service performs face verification operation and returns the personal information of the identified person. If the frame is sent by the camera at the entrance, the system must establish whether the detected person is authorized to get into the office. If the person is not recognized, the system notifies it to the company administration department. NLP subsystem acts when an expected guest has been detected by the facial recognition subsystem. It welcomes the guest, asks the name and the reason for the visit. Then it indicates the direction for the meeting point or notifies administration area of the arrival of the guest.

\section{FINAL REMARKS AND FUTURE WORKS}

In this paper, we presented a smart office management system that leverages IoT and AI techniques to provide several functionalities aiming at optimizing environmental conditions while increasing the wellness of workers, and consequently the company's performances. Future works focus on definitively test and validate the proposed system, even on large scale (more users in a wider workplace), also providing full integration with existing control systems, moving towards the so-called intelligent pervasive spaces [Liu et al. 2010]. In this phase we will evaluate how to include trust issues [Buzzanca et al. 2017] and use a social IoT approach [Carchiolo et al. 2019].

\section{REFERENCES}

Alberdi et al. Using smart offices to predict occupational stress, International Journal of Industrial Ergonomics, Volume 67, 2018, Pages 13-26, ISSN 0169-8141, https://doi.org/10.1016/j.ergon.2018.04.005.

Akbar et al. Contextual occupancy detection for smart office by pattern recognition of electricity consumption data. In: 2015 IEEE ICC, pp. 561-566. http://dx.doi.org/10.1109/ICC.2015.7248381. http://ieeexplore.iee.org/document/ $7248381 /$

Atzori et al., The internet of things: A survey, Computer Networks, vol. 54, no. 15, pp. 2787 2805, 2010. [Online]. Available: http://www.sciencedirect.com/science/article/pii/S1389128610001568

Basnayake et al. Artificial intelligence based smart building automation controller for energy efficiency improvements in existing buildings, Int. J. Adv. Inf. Sci. Technol. 40 (40) (2015)

Bax Energy, 2019. Available: https://www.baxenergy.com/ 
Bolchini et al., Smart buildings: A monitoring and data analysis methodological framework, Building and Environment, vol. 121, pp. 93 105, 2017.

Buzzanca, M., Carchiolo, V., Longheu, A., Malgeri, M., and Mangioni, G. (2017). Direct trust assignment using social reputation and aging. Journal of Ambient Intelligence and Humanized Computing, 8(2):167-175.

Carchiolo, V., Longheu, A., Malgeri, M., and Mangioni, G. (2019). A social inspired broker for m2m protocols. In COMPLEXIS 2019 - Proceedings of the 3rd Intl. Conference on Complexity, Future Information Systems and Risk.

Carchiolo et al. Using Twitter data and sentiment analysis to study diseases dynamics ITBAM 2015, 26th DEXA Conferences and Workshops, http://dx.doi.org/10.1007/978-3- 319-22741-2-2- Springer International Publishing

Chen et al., "The design and implementation of a smart building control system" in 2009 IEEE International Conference on e-Business Engineering, Oct 2009, pp. 255262

Choi et al., Smart office energy-saving service using bluetooth low energy beacons and smart plugs. 2015 IEEE Intl Conf on Data Science and Data Intensive Systems. pp. 247251. http://dx.doi.org/10.1109/DSDIS.2015.69.

Diakopoulos, N.A., Shamma, D.A.: Characterizing debate performance via aggregated twitter sentiment. In: Proceedings of the SIGCHI. pp. 11951198. CHI 10, ACM, New York, NY, USA (2010), http://doi.acm.org/10.1145/1753326.1753504

EU, European Agency for Safety and Health at Work, "Campaign guide: Managing stress and psychosocial risks at work" 2018. Available: https://eguides.osha.europa.eu/stress/GB-EN/

Goncalves et al. Comparing and combining sentiment analysis methods, in Proc. of the First ACM Conf. on Online Social Networks. New York, NY, USA: ACM, 2013, pp. 2738. [Online]. Available: http://doi.acm.org/10.1145/2512938.2512951

Hart, S.G., Staveland, L.E., 1988. Development of NASA-tlx: Results of Empirical and Theoretical Research. pp. 139183. http://dx.doi.org/10.1016/S0166- 4115(08)62386-9. URL.

Jackson and I. Moulinier, NLP for Online Applications: Text Retrieval, Extract and Categ, 2nd ed. John Benjamins, 2007.

Kiyokawa et al. A context-aware multi-modal smart office chair in an ambient environment. In: 2012 IEEE Virtual Reality (VR). IEEE, pp. 14. http://dx.doi.org/10.1109/VR.2012.6180951. URL. http://ieeexplore.ieee.org/ document/6180951/.

Lake et al. "Building machines that learn and think like people", Behavioral and Brain Sc., vol. 40, p. e253, 2017.

Lang, P., 1980. Behavioral Treatment and Bio-behavioral Assessment: Computer Applications. Ablex., Norwood, NJ

Lilis et al. Towards the next generation of intelligent building: An assessment study of current automation and future IoT based systems, Sustainable Cities and Society, Vol. 28, 2017, Pages 473-481, ISSN 2210-6707

Lin et al., "A survey on internet of things: Architecture, enabling technologies, security and privacy, and applications", IEEE Internet of Things Journal, vol. 4, no. 5, pp. 11251142, Oct 2017.

Liu et al. 2010. Pervasive informatics: theory, practice and future directions. Intell. Build. Int. 2, 519.

McDuff, et al. AffectAura: an intelligent system for emotional memory. In: Proceedings of the 2012 ACM CHI pp. 849. http://dx.doi.org/10.1145/2207676.2208525. URL. http://dl.acm.org/citation.cfm?doid=2207676.2208525.

Matoušek, "Description and analysis of IEC 104 Protocol", 2017. http://www.fit.vutbr.cz/research/ pubs/tr.php?file $=\% 2 F p u b \% 2 F 11570 \% 2 F T R I E C 104$.pdf\&id $=11570$

Medhat et al., Sentiment analysis algorithms and applications: A survey, Ain Shams Eng. Journal, vol. 5, no. 4, 2014.

Muoz et al. An Emotion Aware Task Automation Architecture Based on Semantic Tech. for Smart Offices. Sensors (2018).

Nguyen et al. 2014. A review on simulation-based opt. methods applied to building perf. an. Appl. Energy 113, 10431058.

Plageras et al. Efficient IoT-based sensor BIG Data collectionprocessing and analysis in smart buildings, Future Generation Computer Systems, Volume 82, 2018, Pages 349-357, ISSN 0167-739X, https://doi.org/10.1016/j.future.2017.09.082.

Rottondi et al. An energy management service for the smart office. Energies 8 (10), 1166711684. http://dx.doi.org/10.3390/en81011667. URL. http://www.mdpi. com/1996-1073/8/10/11667/

Shaikh et al., Intelligent multi-objective optimization for building energy and comfort management”, Journal of King Saud University - Engineering Sciences, vol. 30, no. 2, pp. 195 204, 2018.

Stankevic et al. Web-based biometric computer mouse advisory system to analyze a User's emotions and work productivity. Eng. Appl. Artif. Intell. 24 (6), 928945. http://dx.doi.org/10.1016/j.engappai.2011.04.006.

Yao et al., "The Analysis and Research of OPC XML-DA Server". Energy Procedia, Vol. 16, Part C, 2012, ISSN 1876-6102, https://doi.org/10.1016/j.egypro.2012.01.240.

Zeiler et al. Visualizing and Understanding Convolutional Networks. In: Fleet D., Pajdla T., Schiele B., Tuytelaars T. (eds) Computer Vision ECCV 2014. ECCV 2014. Lecture Notes in Computer Science, vol 8689. Springer, Cham

Zheng Yi, Evaluation and Implementation of Convolutional Neural Networks in Image Recognition, Journal of Physics: Conference Series, Volume 1087, Machine Learning and Artificial Intelligence, 2018 